\title{
Dynamics of a SQUID ratchet coupled to a nanomechanical resonator
}

\author{
Stefano Pugnetti, ${ }^{1}$ Yaroslav M. Blanter, ${ }^{2}$ Fabrizio Dolcini, ${ }^{1}$ and Rosario Fazio ${ }^{1}$ \\ ${ }^{1}$ NEST-CNR-INFM and Scuola Normale Superiore, Piazza dei Cavalieri 7, I-56126 Pisa, Italy \\ ${ }^{2}$ Kavli Institute of Nanoscience, Delft University of Technology, Lorentzweg 1, 2628 CJ Delft, The Netherlands
}

(Received 9 January 2009; revised manuscript received 18 March 2009; published 11 May 2009)

\begin{abstract}
We investigate the dynamics of a superconducting rectifying circuit, namely, a three-junction superconducting quantum interference device (SQUID), where one arm of the superconducting loop consists of a nanomechanical resonator. We find that the dc characteristic curve of the system displays features that are directly related to the frequency and amplitude of the mechanical oscillations; these effects can be further enhanced by biasing the SQUID with an ac current. We discuss potential future developments of this idea in the field of ultrasensitive position detection, and assess their feasibility in current setups.
\end{abstract}

DOI: 10.1103/PhysRevB.79.174516

PACS number(s): 85.85. $+\mathrm{j}, 85.25 . \mathrm{Dq}, 05.45 .-\mathrm{a}$

\section{INTRODUCTION}

In recent years the research on nanoelectromechanical systems (NEMS) developed as a broad and rapidly growing field. ${ }^{1,2}$ The low mass and small size of NEMS make them excellent candidates for high-precision force, ${ }^{3}$ mass,${ }^{4}$ and position detection, ${ }^{5-10}$ with a sensitivity that is ultimately limited only by quantum mechanics. Measurements on $\mathrm{SiN}$ (Ref. 11) and GaAs (Ref. 12) doubly clamped beams coupled to single-electron transistors have achieved the sensitivity of four times above the standard quantum limit. Moreover, NEMS have been recently envisaged as integrated elements into quantum information circuits, such as one ${ }^{13-15}$ or several ${ }^{16}$ qubits, opening up promising new prospectives also in the field of quantum information processing. To detect the position of tiny mechanical resonators two different techniques are currently applied: optical detection based on coupling of an oscillator to a microwave cavity ${ }^{17-24}$ is very sensitive, although somewhat difficult to integrate in nanocircuits. On the other hand, traditional electrical detection ${ }^{25}$ based on the influence of the motion of the oscillator on the circuit offers the advantage that excitation and detection occur in the same circuit. However, this approach leads to lower sensitivity and does not allow an easy discrimination between electronic and mechanical excitations.

Importantly, most of existent detection schemes are typically based on high-frequency measurements. Quantum effects can be detected at frequencies $\hbar \omega \gtrsim k_{B} T$, which, at present refrigerator temperatures, can be of the order of several GHz. Electrical measurements at such high frequencies usually involve ad hoc techniques to filter signals, to account for the environment impedance and back action, and to analyze the spectrum. ${ }^{26,27} \mathrm{~A}$ crucial issue is therefore whether it is possible to conceive procedures that are based on dc measurements. In this paper we address this problem. Our idea is based on the so-called ratchet effect. ${ }^{28}$ In very simple terms the ratchet effect can be explained by considering a particle moving in an external periodic potential and subject to a periodic (or random) force with zero average. If the potential breaks spatial inversion symmetry, a directed motion is possible even if the system is unbiased. By detecting the directed motion of a particle it is in general possible to gain information on the oscillating force. Here we discuss in de- tails the case when the nanomechanical resonator is a part of a superconducting quantum interference device (SQUID) loop. It is well known that SQUIDs are currently used as ultrasensitive magnetic field detectors, and that they represent a solid-state implementation of qubits based on Josephson effect. ${ }^{29}$ A remarkable attention in the last few years has also been devoted to coupling SQUIDs to mechanical oscillators for detection or cooling ${ }^{3,14,30-32}$ and, very recently, the first detection of mechanical motion of a micromechanical resonator embedded in a dc SQUID was reported. ${ }^{33}$

It has been shown ${ }^{34}$ that SQUIDs can behave as ratchets, where the superconducting phase difference at the junction $\gamma$ and the Josephson potential play the role of the coordinate and the ratchet potential, respectively. When such systems are ac biased, a constant drift in the phase drop occurs under appropriate conditions, causing a finite dc voltage.

In this work we propose to exploit the rectifying properties of these SQUID ratchets in order to characterize the motion of a nanomechanical resonator; indeed a mechanical oscillator embedded in a SQUID circuit threaded by a magnetic flux produces an ac signal across the SQUID that plays the role of a zero-average driving force, affecting the dc characteristic of this ratchet circuit. We find that the currentvoltage curve is qualitatively changed by the mechanical oscillations with respect to the case without a moving part; in principle certain features can be extracted that can only be attributed to the presence of mechanical oscillations, such as Shapiro steps and a nonmonotonic dip-and-peak structure in the symmetric part of the curve coming from them. This result may in principle pave the way to new detection schemes that circumvent the difficulties related to highfrequency regimes.

The paper is organized as follows. In Sec. II we introduce the model and discuss the physically relevant scales. The results for the current-voltage characteristics are then presented; depending on physical parameters of the junctions employed, the adiabatic limit of a slowly oscillating resonator may be of relevance and it is presented in Sec. III. However, higher coupling efficiency may require slower time scales for the motion of the resonator, thus necessitating a more general discussion for arbitrary resonator frequency, which is done in Sec. IV. We discuss the role of thermal fluctuations in Sec. V, and we then proceed with a critical assessment of the experimental realization of this scheme in 

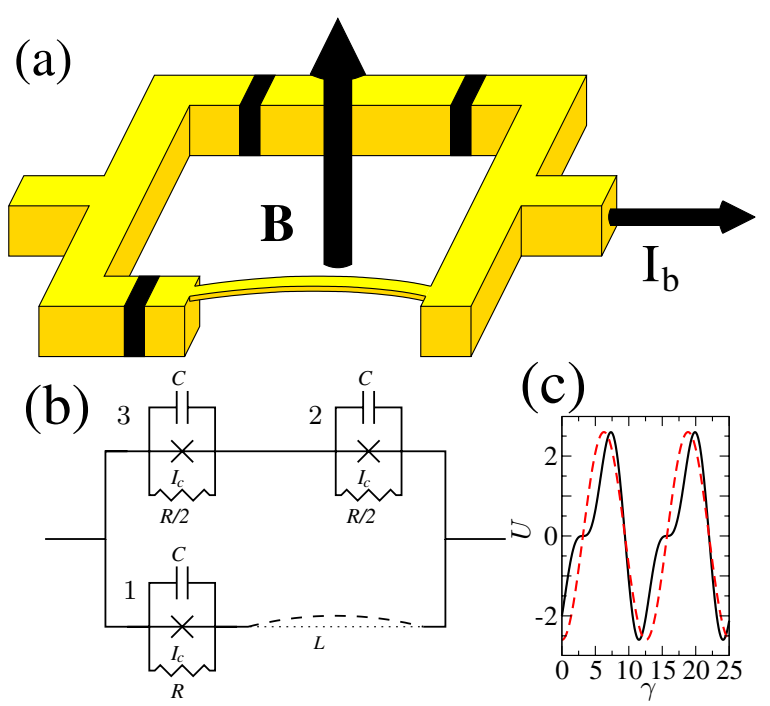

FIG. 1. (Color online) (a)-(b) A model and the corresponding scheme of the ratchet circuit considered here. Three Josephson junctions are coupled to a mechanical resonator oscillating in the plane of the circuit, threaded by a magnetic flux, and biased by a current. The junctions have equal critical currents and capacitances but different shunting resistances. (c) The potential $U(\gamma)$ in the absence of a moving part (solid line); the average slope in the decreasing part is larger in modulus than the average slope of the increasing part, as can be seen by comparing with a cosine curve (dashed line).

existing setups (Sec. VI) by comparing the numerical values of the relevant physical parameters. Concluding remarks and the description of a characterization technique for NEMS are presented in Sec. VII.

\section{THE MODEL}

The circuit that we analyze is schematically depicted in Fig. 1. It consists of a Josephson junction (JJ) and a mechanical resonator connected in parallel with two other JJs. The Josephson junctions are in the classical regime and they can be described with the RCSJ model, i.e.,

$$
\frac{I_{i}}{I_{c}}=\sin \gamma_{i}+\frac{\hbar}{2 e R_{i} I_{c}} \dot{\gamma}_{i}+\frac{\hbar C}{2 e I_{c}} \ddot{\gamma}_{i}, \quad i=1,2,3 .
$$

In Eq. (1) the current flowing through each junction is denoted by $I_{i}$, and $\gamma_{i}$ is the related gauge-invariant phase difference. In Eq. (1) $C$ denotes the capacitance, and $R_{i}$ the resistances, chosen as $R_{1}=R$ and $R_{2}=R_{3}=R / 2$ (see Fig. 1). For simplicity we assumed that the three junctions are characterized by the same critical current $I_{c}$. By considering overdamped junctions, $\omega_{\mathrm{pl}} R C \ll 1\left(\omega_{\mathrm{pl}}=\sqrt{2 e I_{c} / \hbar C}\right.$ is their plasma frequency), the terms involving second-order derivatives of the phases can be neglected in Eq. (1). One can then show that, if $\gamma_{2}=\gamma_{3}$ holds at a certain time, such condition is maintained. We shall henceforth set $\gamma_{2}=\gamma_{3}=\gamma / 2$, whereas the phase $\gamma_{1}$ is related to the other ones by the equation

$$
\gamma_{1}-\gamma=2 \pi\left(\frac{\Phi}{\Phi_{0}}+n\right), \quad n=0,1, \ldots
$$

ensuring a vanishing total phase difference along the loop. In the previous equation $\Phi$ denotes the total magnetic flux threading the circuit and $\Phi_{0}$ is the elementary quantum of flux. Neglecting the self-inductance of the loop, the flux is determined by the total area of the circuit, which in turn depends on the position of the resonator: $\Phi=B[A+l X(t)][l$ is the effective length of the resonator, $X(t)$ is the position of its center of mass and $A$ is the area of circuit when $X(t)=0$ ]. In the following we will assume that the motion of the resonator is fixed externally and equals $X(t)=X_{0} \cos (\omega t)$ (see Sec. VI). Indicating by $I_{b}$ the current biasing the device, current conservation $I_{b}(t)=I_{1}(t)+I_{2}(t)$ leads to the following equation of motion for the phase $\gamma$ :

$$
\partial_{\tau} \gamma=-\frac{\partial U}{\partial \gamma}+\frac{I_{b}(\tau)}{I_{c}}+\frac{\pi L B X_{0}}{\Phi_{0}} \Omega \sin (\Omega \tau),
$$

where $\tau=\omega^{*} t$ is a dimensionless time variable, $\omega^{*}=e R I_{c} / \hbar$ and $\Omega=\omega / \omega^{*}$. We will consider external bias of the form $I_{b}(\tau)=I_{\mathrm{dc}}+I_{\mathrm{ac}} \cos \left(\Omega_{\mathrm{ac}} \tau\right)$, with $I_{\mathrm{dc}}$ denoting the dc component, and $I_{\mathrm{ac}}$ and $\Omega_{\mathrm{ac}}$ the amplitude and frequency of a monochromatic ac component. The periodic function

$$
U[\gamma, f(\tau)]=-2 \cos (\gamma / 2)-\cos [\gamma+\phi+f(\tau)]
$$

is the potential leading to the ratchet effect as discussed by Zapata et $a l .{ }^{34}$ In addition to the dimensionless external magnetic-flux $\phi=2 \pi A B / \Phi_{0}$, the potential $U$ now includes also a time-dependent fluctuation part $f(\tau)$ $=\left(2 \pi L B X_{0} / \Phi_{0}\right) \cos (\Omega \tau)$ which arises from the oscillations of the mechanical resonator. The coupling between the SQUID and the mechanical resonator can be parametrized by the dimensionless parameter

$$
a=\frac{2 \pi L B X_{0}}{\Phi_{0}} .
$$

At finite temperatures the rhs of Eq. (3) will also include a noise term.

Depending on the value of the external flux, the potential term defined in Eq. (4) breaks $\gamma$ inversion symmetry and therefore leads to the ratchet effect. In Ref. 34 this effect has been shown by biasing the SQUID with an oscillating current leading to a finite dc voltage. Here we show that the ratchet effect allows one to detect the oscillatory motion of the oscillator which enters the dynamics of the systems both in the potential through the fluctuating part of the flux and in the third term on the rhs of Eq. (3). We emphasize that, in order for the ratchet effect to appear, the system needs to be out of thermal equilibrium. ${ }^{28}$

The dc voltage drop across the circuit can be expressed through the Josephson relation as

$$
V_{0}=\frac{\hbar}{2 e}\left\langle\frac{d \gamma}{d t}\right\rangle=\frac{R I_{c}}{2}\left\langle\partial_{\tau} \gamma\right\rangle,
$$

where the brackets $\langle\ldots\rangle$ indicate time average (and possibly thermal average). A nonvanishing voltage drop can be obtained when the ratchet potential is asymmetric in $\gamma$, which 

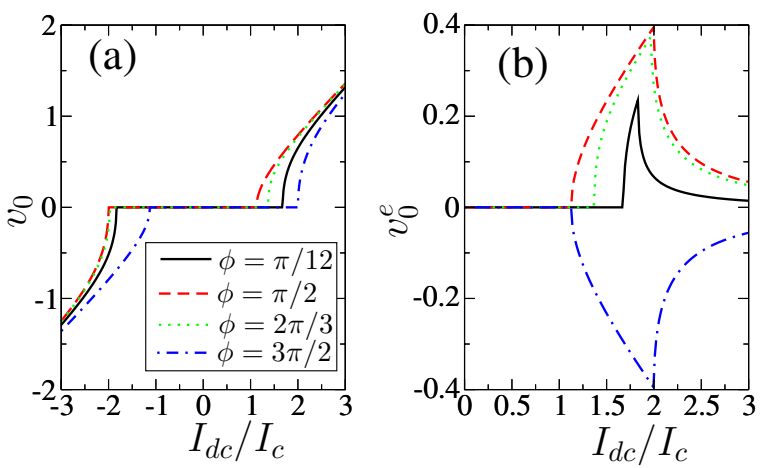

FIG. 2. (Color online) (a) Dimensionless dc voltage drop $v_{0}$ $=V_{0} / R I_{c}$ as a function of the dimensionless bias current $I_{\mathrm{dc}} / I_{c}$, for different values of the flux. No oscillating mechanical part is present $(a=0)$. (b) The even part $v_{0}^{e}$ of the same curves plotted for positive values of $I_{\mathrm{dc}}$ only. $v_{0}^{e}$ is zero for $\phi=n \pi$ and $v_{0}^{e}(-\phi)$ $=-v_{0}^{e}(\phi)$.

occurs for values of the magnetic-flux $\phi \neq n \pi$. In particular one finds that, for sufficiently large values of $I_{\mathrm{ac}}$, a finite dc voltage $V_{0} \neq 0$ arises even in the presence of a vanishing average drive $\left(I_{\mathrm{dc}}=0\right)$. Furthermore, for $I_{\mathrm{dc}} \neq 0$ the dc $V-I$ curve is not an odd function of the current. It is therefore convenient to analyze its symmetric (even) part defined as

$$
v_{0}^{e} \doteq \frac{V_{0}\left(I_{\mathrm{dc}}\right)+V_{0}\left(-I_{\mathrm{dc}}\right)}{2 R I_{c}} .
$$

Mechanical oscillations lead to two effects in the dynamics of the SQUID. On one hand a term qualitatively similar to an ac bias arises, as shown by the last term on the rhs of Eq. (3). Second, a fluctuating term $f(\tau) \neq 0$ appears in the potential, affecting the symmetry of the ratchet potential as a function of $\gamma$. In the presence of the resonator the analysis is thus more subtle. As we shall see in Sec. III, the fluctuations of the potential tend to wash out the ratchet effect; although for some specific values of the parameters one can still obtain $V_{0}\left(I_{\mathrm{dc}}=0\right) \neq 0$. In most cases the ratchet effect survives only in some nontrivial features in the symmetric part of the $V$-I curve, which we will mainly focus on in the rest of the paper.

Apart from some specific cases the equation of motion of the SQUID [Eq. (3)] does not allow for an analytic solution. Most of our results are based on a numerical integration of the equation of motion. As we are interested in using the SQUID as a detector of the mechanical oscillations, it is appropriate to first briefly discuss the case where no oscillator is present, in order to highlight the difference due to the mechanical motion. When the SQUID is biased by a dc current $\left[I_{b}(\tau)=I_{\mathrm{dc}}\right]$ and there is no resonator $(a=0)$ the voltage drop can straightforwardly be computed as $V_{0}$ $=4 \pi\left(R I_{c} / 2\right) / T$, where $4 \pi$ is the period of potential (4), and

$$
T=\int_{0}^{4 \pi} \frac{d \gamma}{I_{\mathrm{dc}} / I_{c}-\sin (\gamma / 2)-\sin (\gamma+\phi)}
$$

is the time required for the phase to span such an angle. The resulting $V$ - $I$ curve of the device is shown in Fig. 2(a). For an asymmetric potential $(\phi \neq n \pi)$ the curves are not odd in the current, and a nonvanishing even component (7) arises [see Fig. 2(b)]. This component will be shown to be affected by the presence of the mechanical resonator. As the flux varies, the curve $v_{0}\left(I_{\mathrm{dc}}\right)$ shifts to the left and then to the right with a period of $2 \pi$; this reflects in the amplitude and sign of $v_{0}^{e}$. Indeed, from Eq. (8) one can show that $v_{0}^{e}(-\phi)=-v_{0}^{e}(\phi)$ and $v_{0}^{e}(\phi=0)=0$.

\section{SLOWLY OSCILLATING RESONATOR-THE ADIABATIC LIMIT}

We now discuss the role of the resonator. Before addressing the full numerical solution of Eq. (3), we discuss here the limiting situation in which $\Omega \ll 1$, i.e., the oscillator's frequency is much smaller than the plasma frequency of the SQUID. As we shall see, this regime is indeed suggested by the typical experimental conditions, where $\Omega \sim 10^{-3}$, indicating that the mechanical oscillations are much slower than the dynamics of the junctions. Moreover the same condition can be realized for the frequency of the ac component of the bias current. Under these conditions, the equation of motion can be solved within the adiabatic approximation. One can determine $v_{0}\left(I_{\mathrm{dc}}\right)$ by evaluating, for a fixed position of the oscillator $X(\tau)$ and value of the bias current $I_{b}(\tau)$, the time $T(\tau)$ required for the phase to span an angle $4 \pi$. Averaging over $\tau$ one then obtains

$$
\begin{aligned}
v_{0} & =\frac{1}{2}\langle 4 \pi / T(\tau)\rangle \\
& =\int_{0}^{2 \pi}\left[\int_{0}^{4 \pi} \frac{d \gamma}{I_{b}(\theta) / I_{c}-\partial_{\gamma} U[\gamma, f(\theta)]+\frac{a \Omega}{2} \sin (\theta)}\right]^{-1} d \theta .
\end{aligned}
$$

Equation (9) will be analyzed in two different cases in which the bias current is constant or has also an additional monochromatic ac component.

\section{A. dc bias current}

We start by analyzing the situation of a dc bias current, $I_{b}(\tau)=I_{\mathrm{dc}}$. In Figs. 3(a) and 3(b) even component (7) of the voltage is plotted as a function of $I_{\mathrm{dc}}$, for different values of the coupling strength $a$ and flux $\phi$. For small coupling $a$ $\ll 1$, we recover the exact results discussed in Sec. II [see Fig. 2(b)]. On the other side, at high values of the coupling the main effect of the mechanical oscillations is to wash out the effects of the asymmetry of the ratchet potential. This is due to the fact that in this system the resonator oscillations also affect the ratchet potential via the term $f(t)$. Notice that the disappearance of asymmetry occurs at lower values of $a$ for a smaller flux $\phi$, as one can see by comparing the dashed curves in Figs. 3(a) and 3(b). Thus the effects of the coupling to the mechanical resonator are more dramatic for $\phi \sim n \pi$. Note, however, that for the special values $\phi=n \pi$, where $n$ is an integer, one still finds $v_{0}^{e}=0$, as can be shown by a change in integration variables $\gamma \rightarrow-\gamma$ and $\theta \rightarrow \pi+\theta$ in Eq. (9) with $I_{b}(\tau) \equiv I_{\mathrm{dc}}$. We notice also that the experimentally accessible values of $a$ are very small. Although in Sec. VI possible 

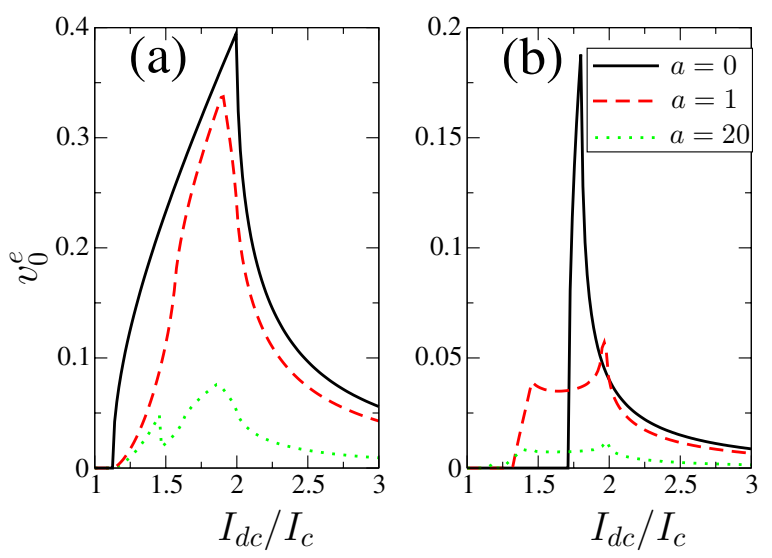

FIG. 3. (Color online) $v_{0}^{e}\left(I_{\mathrm{dc}}\right)$ curves for different values of the coupling parameter $a$. The two plots refer to different values of the flux: (a) $\phi=\pi(n+1 / 2)$ and (b) $\phi=\pi(n+1 / 20)$. While for small coupling one recovers the curves of Fig. 2, for high coupling the features are washed out. The effect is more pronounced for small flux bias.

operative ways to enhance the coupling $a$ will be proposed, these results lead to conclude that, in the presence of a purely dc bias $I_{\mathrm{dc}}$, it is difficult to gain information on a realistic mechanical resonator. A possible way out is to inject current with a monochromatic ac component with frequency $\omega_{\mathrm{ac}} / 2 \pi$, small enough for the adiabatic approximation still to hold. This is discussed in the next subsection.

\section{B. ac monochromatic bias}

In this case some new effects that can be clearly attributed to the mechanical resonator indeed arise. One might naively expect that such effects simply originate from resonances at specific values of $\omega_{\mathrm{ac}}$ related to $\omega$, yielding an enhancement of the effects due to the mechanical oscillations. However, as we shall discuss below, the situation is more complex and the overall features of the current-voltage characteristics originate from different mechanisms.

We assume $I_{b}(\tau)=I_{\mathrm{dc}}+I_{\mathrm{ac}} \cos \left(\Omega_{\mathrm{ac}} \tau\right) \quad\left(\Omega_{\mathrm{ac}}=\omega_{\mathrm{ac}} / \omega^{*}\right)$ and we restrict to the case $\Omega_{\mathrm{ac}}=\Omega q / p$, with $q$ and $p$ integers with no common divisor apart from 1. A straightforward calculation allows to rewrite Eq. (9) as

$$
\begin{aligned}
v_{0}= & \int_{0}^{2 \pi} d \theta\left\{\int _ { 0 } ^ { 4 \pi } d \gamma \left[\frac{I_{\mathrm{dc}}}{I_{c}}+\frac{I_{\mathrm{ac}}}{I_{c}} \cos (q \theta)-\sin \left(\frac{\gamma}{2}\right)\right.\right. \\
& \left.\left.-\sin [\gamma+\phi+a \cos (p \theta)]+\frac{a \Omega}{2} \sin (p \theta)\right]^{-1}\right\}^{-1}
\end{aligned}
$$

and to determine how the ac bias affects the $V-I$ curves of Fig. 3. The result is shown in Fig. 4 for two different values of the flux.

The even component $v_{0}^{e}$ is suppressed for strong coupling, similarly to the dc bias case. However, a richer structure due to the interplay of the two components of the driving bias is observable. Another noteworthy difference with respect to the dc case is that, at values of the dimensionless flux close to $n \pi$, the suppression occurs at higher values of the param-
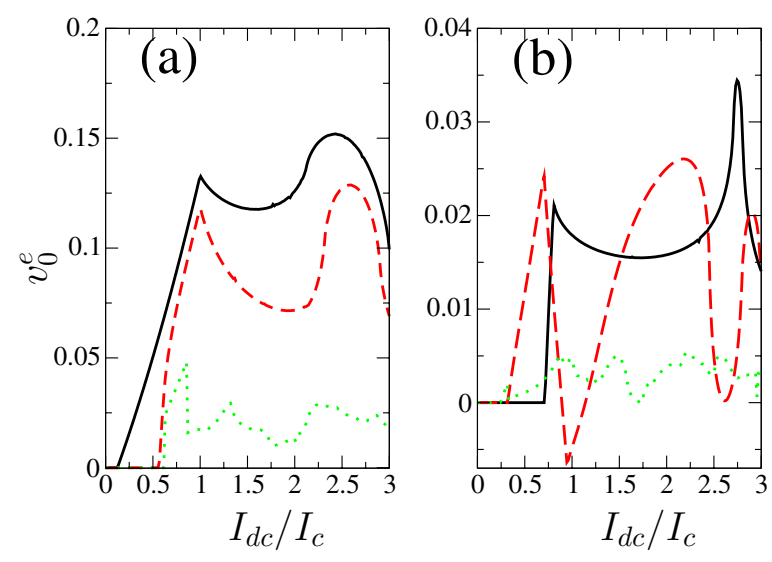

FIG. 4. (Color online) $v_{0}^{e}\left(I_{\mathrm{dc}}\right)$ curves for $I_{\mathrm{ac}}=I_{c}, \Omega_{\mathrm{ac}}=2 \Omega / 3$ and different values of the coupling parameter $a$ (the same as in Fig. 3). The two plots refer to different values of the flux bias: (a) $\phi$ $=\pi(n+1 / 2)$ and (b) $\phi=\pi(n+1 / 20)$. Again for small coupling the curves are indistinguishable from the uncoupled ones $(a=0)$; however for high couplings now a richer structure appear and its set on occurs at a smaller coupling for $\phi \sim n \pi$.

eter $a$, as one can observe by comparing the curves of Fig. 4(b) with Fig. 3(b). This indicates that the presence of an ac current makes the even component of the characteristic curve much more robust to the coupling with the resonator. Such difference is particularly striking at $\phi=n \pi(n=0, \pm 1, \ldots)$. For these particular values of flux $v_{0}^{e}$ vanishes for a purely dc bias current, whereas a finite value of $v_{0}^{e}$ is predicted for an ac current. Indeed the argument used to prove that $v_{0}^{e} \equiv 0$ if $\phi=n \pi$ (a suitable change in the integration variables) does not apply when $I_{\mathrm{ac}} \neq 0$, unless the frequency assumes specific values, namely, those for which $p$ and $q$ are both odd integers. One can see the effects of the two combined oscillations in Fig. 5. Notice that these curves cannot be due to an ac bias alone, for if $a=0$ and $I_{\mathrm{ac}} \neq 0$ the same argument of the previous section would apply, and the $V-I$ curve at $\phi$ $=n \pi$ would be odd .

These results suggest a possible operative detection protocol for the tiny motion of a mechanical resonator integrated

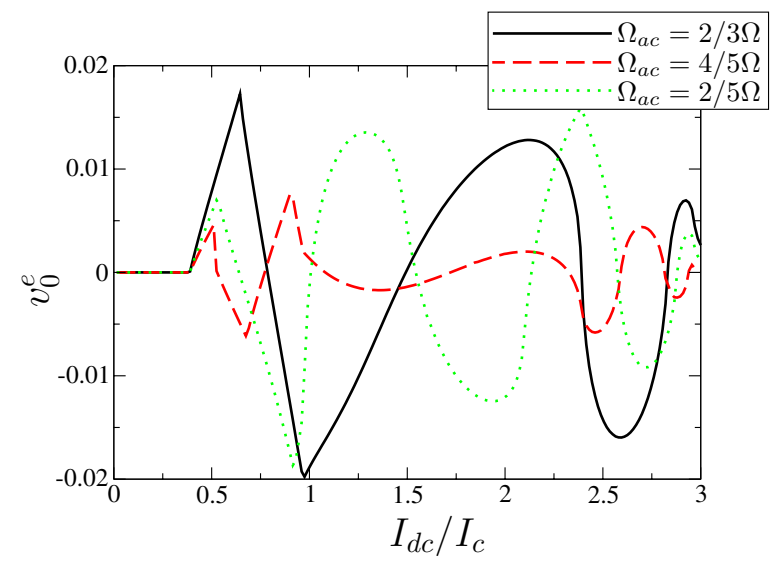

FIG. 5. (Color online) $v_{0}^{e}\left(I_{\mathrm{dc}}\right)$ for zero bias flux and different values of the ratio $\Omega_{\mathrm{ac}} / \Omega$. Here $a=1$, while for $a=0$ the curves should be flat $\left(v_{0}^{e} \equiv 0\right)$. 

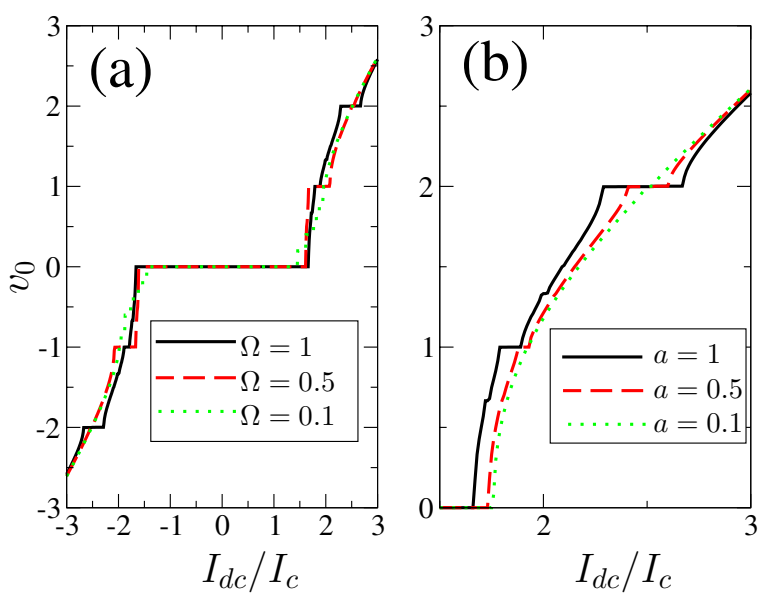

FIG. 6. (Color online) (a) Exact $v_{0}-I_{\mathrm{dc}}$ curves for different frequencies $\Omega$ of the mechanical resonator. Here $a=1, I_{\mathrm{ac}}=0$ and $\phi$ $=n \pi$. Even though no ac bias current is supplied, the system exhibits Shapiro steps, whose heights are related to $\Omega$. (b) The same but for a fixed frequency $\Omega=1$ and different values of the coupling $a$. The width of Shapiro steps approaches zero for small values of $a$.

in a SQUID ratchet circuit: one may first measure the $V-I$ curve of the circuit biased by a purely dc current, for different values of magnetic-flux $\phi$. This would allow to determine values of $\phi$ such that the $V$ - $I$ curve is completely odd. Then, by adding an ac term to the bias current, without changing the magnetic field, a nonvanishing even component would arise in the $V-I$ curve with varying the frequency of the ac bias.

\section{ARBITRARY RESONATOR FREQUENCY}

In many situations the value of the coupling parameter $a$ is rather small (see Sec. VI); for improving the coupling efficiency one then could in principle study devices with a higher value of the frequency $\Omega$, so that the coupling term $a \Omega \cos (\Omega \tau) / 2$ can be increased. Under these circumstances the adiabatic approximation may not hold anymore and one should compute the current-voltage characteristic starting from the complete numerical solution of Eq. (3).

The results are shown in Fig. 6(a), where the $V$ - I curves at strong coupling are plotted, in the absence of the ac bias current, at vanishing flux and for different values of the dimensionless frequency $\Omega$. One can clearly recognize the typical shape of the $V$-I curve for ac biased Josephson junctions, characterized by Shapiro steps. However, while these features are usually attributed to an ac bias current, here they originate from the mechanical oscillations. Notice that the height of the steps varies with the proper frequency of the resonator, as it happens for Shapiro steps in isolated Josephson junctions. This suggests that in principle the frequency of the resonator can be directly read out by inspection of the height of the steps in the $V-I$ curve of the system. The width of the steps, instead, is usually related to the amplitude $a$ of the ac biasing signal. This is illustrated in Fig. 6(b), where one can see that, as $a$ approaches zero, the steps become narrower and the $V-I$ curve becomes smoother.

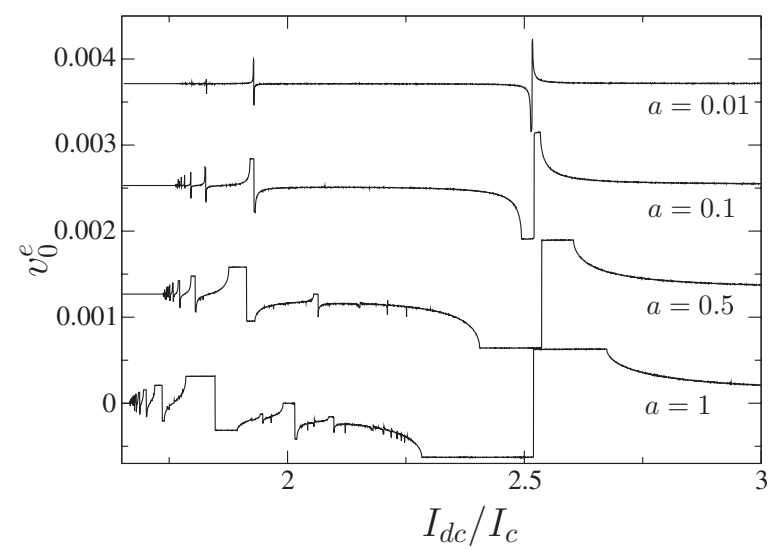

FIG. 7. The even component $v_{0}^{e}$ of the voltage drop as a function of the dc bias current $I_{\mathrm{dc}}$ at $\phi=n \pi$ and $\Omega=1$ for different values of the coupling $a$ (the curves are displaced vertically). The amplitude of the features is the same for all couplings, but their widths become smaller for smaller couplings.

Important differences emerge with respect to the adiabatic limit. In particular the even component of the $V$ - $I$ curve does not vanish for $\phi=n \pi$ and $I_{\mathrm{ac}}=0$, and exhibits an interesting structure: ranges where the voltage is independent of $I_{\mathrm{dc}}$ are separated by sequences of maxima and minima, as shown in Fig. 7. Interestingly, the amplitude characterizing these features in the even component of the $V-I$ curve seems roughly independent of the coupling parameter $a$. This represents a promising effect for the detection of realistic mechanical oscillators. However, as it is already the case for the whole curve $v_{0}\left(I_{\mathrm{dc}}\right)$ (Fig. 6), the width of the different peaks is suppressed for small coupling.

The effect of an ac bias is to enhance the amplitude of these features; indeed the ratio between the heights of the features in Fig. 8 and those in Fig. 7 is about 50. The numerical solution indicates that, in the absence of a mechanical oscillator $(a=0)$, the $V-I$ curve is odd $\left(v_{0}^{e} \equiv 0\right)$, as in the adiabatic limit. We conclude that at $\phi=n \pi$ a detected signal has to be attributed to the mechanical resonator only. Even though the main difficulty at low coupling still remains, these effects may be enhanced by applying an ac bias.

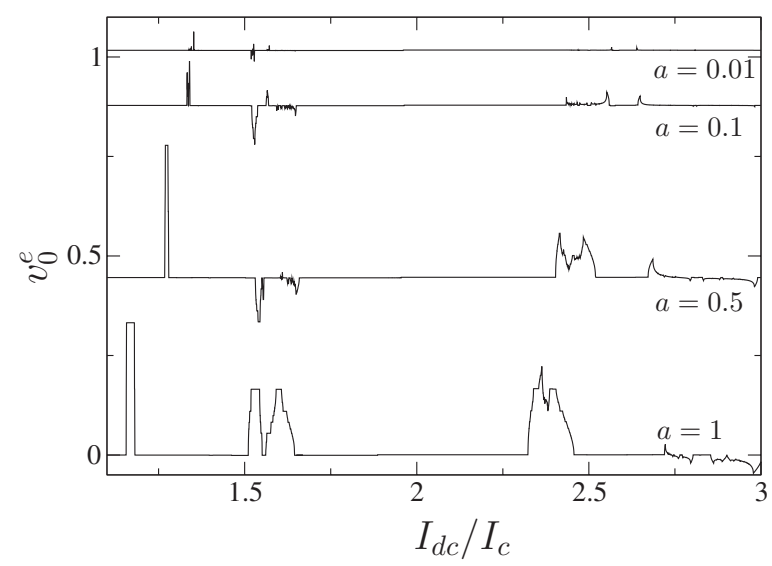

FIG. 8. The even component $v_{0}^{e}$ of the voltage drop as a function of the dc bias current $I_{\mathrm{dc}}$ at $\phi=n \pi$ and $I_{\mathrm{ac}}=I_{c}$, for different values of the coupling $a$. Here $\Omega=1$ and $\Omega_{\mathrm{ac}}=0.67$. 


\section{FINITE TEMPERATURES}

In this section we discuss the effects of thermal fluctuations on the dc voltage drop of the system. To be consistent with the model adopted so far, we shall focus on the classical regime and account for finite temperature by adding a stochastic term (white noise) in Eq. (3). These fluctuations are associated to two sources of dissipation: the finite resistance $R / 2$ of the circuit, giving rise to electrical fluctuations in the bias current, and the finite quality factor $Q$ of the mechanical resonator, related to a random force producing mechanical fluctuations. Electrical fluctuations are taken into account by adding a term

$$
\Delta I_{b}(\tau)=I_{c} \sqrt{\frac{4 e k_{B} T}{\hbar I_{c}}} \xi(\tau),
$$

to the bias current $I_{b}(\tau)$, where $\xi(\tau)$ is a white-noise process with zero average and correlation function $\left\langle\xi(\tau) \xi\left(\tau^{\prime}\right)\right\rangle$ $=\delta\left(\tau-\tau^{\prime}\right)$. Mechanical fluctuations may be introduced via a stochastic force,

$$
F(t)=\sqrt{\frac{2 m \omega k_{B} T}{Q}} \eta(t),
$$

acting on the resonator. Here $m$ is the mass of the mechanical oscillator and $\eta(t)$ another white-noise process, statistically independent of $\xi$. In the previous sections we assumed the motion of the mechanical oscillator to be undamped $(Q$ $=\infty$ ), so that the mechanical noise should be neglected. However for realistic devices this treatment is still a good approximation if

$$
k_{B} T \ll Q \frac{1}{2} m \omega^{2} x_{0}^{2},
$$

with $x_{0}$ denoting the amplitude of the oscillation. For high $Q$ there is a regime in which temperature is low enough to fulfill condition (13), but high enough to fulfill $k_{B} T \gg \hbar \omega$, so that the behavior is neither quantum nor noisy. In the following we shall assume that the mechanical noise can be neglected, and analyze Eq. (3) with

$$
I_{b}(\tau)=I_{\mathrm{dc}}+I_{\mathrm{ac}} \cos \left(\Omega_{\mathrm{ac}} \tau\right)+I_{c} \sqrt{\Theta} \xi(\tau),
$$

where $\Theta=T / T_{0}$ and $T_{0}=\hbar I_{c} / 4 e k_{B} \simeq 12 \mathrm{~K} I_{c} / \mu \mathrm{A}$. As one may expect, for a certain value of the coupling parameter $a$ there is a value of $\Theta$ above which the peculiar features of the characteristic curve are lost. For determining the temperature scale at which this happens, one has to know $T_{0}$, which is set by the value of the critical current. Devices with large critical currents are in principle the best for this detection scheme, since they will be less affected by temperature. For a critical current as large as $1 \mathrm{~mA}$, the temperature scale is $10^{4} \mathrm{~K}$. As an example, consider Fig. 9, which is a detail of the $v_{0}^{e}\left(I_{\mathrm{dc}}\right)$ curve in Fig. 8 for $a=0.1$, compared with its finite temperature counterpart. Thermal fluctuations tend to wash out the peculiar features of the dc characteristic; the temperature scale at which this happens is $0.1 \mathrm{mK}$ for a high- $I_{c}$ device, corresponding to $\Theta \approx 10^{-8}$.

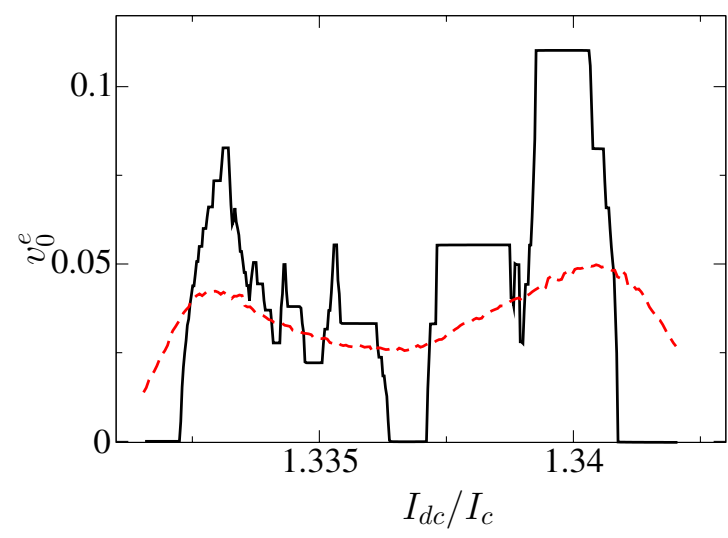

FIG. 9. (Color online) Some details of Fig. 8 for $a=0.1, I_{\mathrm{ac}}=1$, $\Omega=1$, and $\Omega_{\mathrm{ac}}=0.67$; the curves correspond to different temperatures: $\sqrt{\Theta}=0$ (solid line) and $\sqrt{\Theta}=10^{-4}$ (dashed line).

\section{EXPERIMENTAL PERSPECTIVES}

By now top-down fabrication techniques can produce single-crystal doubly clamped beams whose typical dimensions are $1 \mu \mathrm{m} \times 0.1 \mu \mathrm{m} \times 0.1 \mu \mathrm{m}$ and whose frequency ranges from tens of $\mathrm{MHz}$ (Ref. 35) to $1 \mathrm{GHz}$ (Ref. 36); using silicon nitride or carbide, the density is about $3 \times 10^{3}$ $\mathrm{kg} / \mathrm{m}^{3}$ and hence typical masses are of the order of $10^{-17} \mathrm{~kg}$. Lower values can be obtained using carbon nanotubes, which can also have higher oscillation frequencies due to their high bulk modulus. ${ }^{37}$ The oscillation can be induced by driving the resonator either mechanically (with a piezoelement applied on the substrate and driven by an ac signal) or electrically (for instance by capacitively coupling the resonator with a gate electrode); a $1 \mu \mathrm{m}$-long beam can be easily excited up to oscillation amplitudes of $1 \mathrm{~nm}$. To estimate the corresponding coupling parameter $a$ one has to keep in mind that the magnetic field causing the coupling cannot be too large, otherwise the device loses superconductivity; a typical upper bound is $B<0.1 \mathrm{~T}$. With this value, one gets $a \approx 0.1$, the value used for the plots in Fig. 9.

The intensity of the back action on the beam can be estimated by the Lorentz force acting on it in virtue of the current flowing through it: $F_{l}=L I B \simeq 1 \mu \mathrm{m} \times 1 \mathrm{~mA} \times 0.1 \mathrm{~T}$ $=10^{-10} \mathrm{~N}$; this is to be compared with $m \omega^{2} x_{0} \simeq 10^{-17} \mathrm{~kg}$ $\times 4 \pi^{2}(1 \mathrm{GHz})^{2} \times 10^{-9} \mathrm{~m}=4 \times 10^{-7} \mathrm{~N}$. (At such amplitudes the force may be no longer linear in the displacement and it would probably be larger than this estimate.) The back action can thus be neglected as a first approximation.

As for the adiabatic approximation, the value of $\Omega$ $=\hbar \omega / e R I_{c}$ depends on the resistance of the junction. For tunnel junctions the low-temperature resistance is due only to quasiparticle tunneling and increases exponentially with decreasing temperature; at temperatures close to the critical temperature the resistance can be estimated with the normalstate resistance and $I_{c} R_{n}$ can be as large as $1 \mathrm{mV}$, corresponding to $\omega^{*} \simeq 10^{12} \mathrm{~Hz}$. In such situations the adiabatic approximation could apply. However the resistance of a Josephson junction can vary greatly and thus the validity of the adiabatic approximation depends on the device.

\section{CONCLUSIONS}

The dc current-voltage curve of a SQUID ratchet circuit is significantly affected by the motion of a part of the circuit 
and the induced features can be used to characterize a nanomechanical resonator. This curve exhibits Shapiro steps whose heights can be used to determine the frequency of the mechanical oscillations. Once this frequency is known, either directly with this de technique or with others, one can bias the device with an ac signal whose frequency should be chosen in order to improve the effects of the mechanical oscillations; we suggest to use an ac frequency of two-thirds the estimated frequency of mechanical oscillations. Now, by varying the magnetic field through the device, the symmetric part of the characteristic curve should evolve periodically (the period being set by the flux quantum); the amplitude of the curve will be maximum for frustrated values of the flux $\left[\Phi_{e}=(n+1 / 2) \Phi_{0}\right]$ and minimum for unfrustrated values $\left(\Phi_{e}=n \Phi_{0}\right)$. If there were no mechanical oscillations in the circuit, then the symmetric part of the characteristic curve should be zero for unfrustrated flux bias, since the potential experienced by the superconducting phase would be sym- metric; everything that can be seen in this case is due to the interplay between the ac driving field and the signal due to the mechanical oscillations of the integrated nanoresonator. The shape of the characteristic curve can be compared with the finite temperature results we illustrated in this work (see, for instance, Fig. 9). We emphasize that no ac measurement is required for this characterization scheme.

\section{ACKNOWLEDGMENTS}

We acknowledge very fruitful discussions with Herre van der Zant and Menno Poot. The work was supported by SNS05 of Scuola Normale Superiore and by Netherlands Foundation for Fundamental Research on Matter (FOM). S.P. acknowledges the Kavli Institute of Nanoscience for their kind hospitality, and F.D. acknowledges the Italian MIUR "Rientro dei Cervelli" Program for financial support.
${ }^{1}$ A. N. Cleland, Foundations of Nanomechanics (Springer, Berlin, 2003).

${ }^{2}$ A. A. Clerk, M. H. Devoret, S. M. Girvin, F. Marquardt, and R. J. Schoelkopf, arXiv:0810.4729 (unpublished).

${ }^{3}$ M. P. Blencowe and E. Buks, Phys. Rev. B 76, 014511 (2007).

${ }^{4}$ K. L. Ekinci, X. M. H. Huang, and M. L. Roukes, Appl. Phys. Lett. 84, 4469 (2004).

${ }^{5}$ J. D. White, Jpn. J. Appl. Phys., Part 2 32, L1571 (1993).

${ }^{6}$ A. Naik, O. Buu, M. D. LaHaye, A. D. Armour, A. A. Clerk, M. P. Blencowe, and K. C. Schwab, Nature (London) 443, 193 (2006).

${ }^{7}$ M. Li, H. X. Tang, and M. L. Roukes, Nat. Nanotechnol. 2, 114 (2007).

${ }^{8}$ N. E. Flowers-Jacobs, D. R. Schmidt, and K. W. Lehnert, Phys. Rev. Lett. 98, 096804 (2007).

${ }^{9}$ E. Buks, S. Zaitsev, E. Segev, B. Abdo, and M. P. Blencowe, Phys. Rev. E 76, 026217 (2007).

${ }^{10}$ C. A. Regal, J. D. Teufel, and K. W. Lehnert, Nat. Phys. 4, 555 (2008).

${ }^{11}$ M. D. LaHaye, O. Buu, B. Camarota, and K. C. Schwab, Science 304, 74 (2004).

${ }^{12}$ R. G. Knobel and A. N. Cleland, Nature (London) 424, 291 (2003).

${ }^{13}$ P. Rabl, A. Shnirman, and P. Zoller, Phys. Rev. B 70, 205304 (2004).

${ }^{14}$ Y.-D. Wang, K. Semba, and H. Yamaguchi, New J. Phys. 10, 043015 (2008).

${ }^{15}$ J. Hauss, A. Fedorov, S. André, V. Brosco, C. Hutter, R. Kothari, S. Yeshwanth, A. Shnirman, and G. Schön, New J. Phys. 10, 095018 (2008).

${ }^{16}$ X. B. Zou and W. Mathis, Phys. Lett. A 324, 484 (2004).

${ }^{17}$ O. Arcizet, P.-F. Cohadon, T. Briant, M. Pinard, and A. Heidmann, Nature (London) 444, 71 (2006).

${ }^{18}$ S. Gigan, H. R. Bhöm, M. Paternostro, F. Blaser, G. Langer, J. B. Hertzberg, K. C. Schwab, D. Bäuerle, M. Aspelmeyer, and A. Zeilinger, Nature (London) 444, 67 (2006).

${ }^{19}$ M. Poggio, C. L. Degen, H. J. Mamin, and D. Rugar, Phys. Rev. Lett. 99, 017201 (2007)

${ }^{20}$ I. Favero, C. Metzger, S. Camerer, D. König, H. Lorenz, J. P.
Kotthaus, and K. Karrai, Appl. Phys. Lett. 90, 104101 (2007).

${ }^{21}$ M. W. Pruessner, T. H. Stievater, and W. S. Rabinovich, Appl. Phys. Lett. 92, 081101 (2008).

${ }^{22}$ S. Gröblacher, S. Gigan, H. R. Böhm, A. Zeilinger, and M. Aspelmeyer, EPL 81, 54003 (2008).

${ }^{23}$ J. D. Thompson, B. M. Zwickl, A. M. Jayich, F. Marquardt, S. M. Girvin, and J. G. E. Harris, Nature (London) 452, 72 (2008).

${ }^{24}$ A. Schliesser, G. Anetsberger, R. Rivière, O. Arcizet, and T. J. Kippenberg, New. J. Phys. 10, 095015 (2008).

${ }^{25}$ K. R. Brown, J. Britton, R. J. Epstein, J. Chiaverini, D. Leibfried, and D. J. Wineland, Phys. Rev. Lett. 99, 137205 (2007).

${ }^{26}$ G. B. Lesovik and R. Loosen, JETP Lett. 65, 295 (1997); A. A. Clerk, S. M. Girvin, and A. D. Stone, Phys. Rev. B 67, 165324 (2003).

${ }^{27}$ R. J. Schoelkopf, P. J. Burke, A. A. Kozhevnikov, D. E. Prober, and M. J. Rooks, Phys. Rev. Lett. 78, 3370 (1997); B. Reulet, J. Senzier, and D. E. Prober, ibid. 91, 196601 (2003); R. Deblock, E. Onac, L. Gurevich, and L. P. Kouwenhoven, Science 301, 203 (2003); J. Gabelli, L.-H. Reydellet, G. Fève, J.-M. Berroir, B. Plaçais, P. Roche, and D. C. Glattli, Phys. Rev. Lett. 93, 056801 (2004).

${ }^{28}$ P. Reimann, Phys. Rep. 361, 57 (2002).

${ }^{29}$ Y. Makhlin, G. Schön, and A. Shnirman, Rev. Mod. Phys. 73, 357 (2001).

${ }^{30}$ E. Buks and M. P. Blencowe, Phys. Rev. B 74, 174504 (2006).

${ }^{31}$ X. Zhou and A. Mizel, Phys. Rev. Lett. 97, 267201 (2006).

${ }^{32}$ F. Xue, Y. X. Liu, C. P. Sun, and F. Nori, Phys. Rev. B 76, 064305 (2007).

${ }^{33}$ S. Etaki, M. Poot, I. Mahboob, K. Onomitsu, H. Yamaguchi, and H. S. J. van der Zant, Nat. Phys. 4, 785 (2008).

${ }^{34}$ I. Zapata, R. Bartussek, F. Sols, and P. Hanggi, Phys. Rev. Lett. 77, 2292 (1996).

${ }^{35}$ A. N. Cleland and M. L. Roukes, Appl. Phys. Lett. 69, 2653 (1996).

${ }^{36}$ X. M. H. Huang, C. A. Zorman, M. Mehregany, and M. L. Roukes, Nature (London) 421, 496 (2003).

${ }^{37}$ S. Sapmaz, Ya. M. Blanter, L. Gurevich, and H. S. J. van der Zant, Phys. Rev. B 67, 235414 (2003). 